\title{
APOE $\varepsilon 4$ allele modified the correlation between deep grey matter volume and cognitive performance in non-demented elders
}

\author{
Weiping $\mathrm{Li}^{1} \cdot \mathrm{Yu} \mathrm{Xie}^{2} \cdot$ Tingting $\mathrm{Yu}^{2} \cdot$ Wenbo $\mathrm{Wu}^{1} \cdot \mathrm{Kun} \mathrm{Wang}^{1} \cdot$ Sichu $\mathrm{Wu}^{1} \cdot \mathrm{Zhao} \mathrm{Qing}^{1} \cdot \mathrm{Xin}_{\mathrm{Zhang}}{ }^{1} \cdot \mathrm{Ming} \mathrm{Li}^{1}$. \\ Renyuan Liu ${ }^{3} \cdot$ Zhenyu Yin $^{2} \cdot$ Bing Zhang ${ }^{1}$
}

Received: 14 February 2020 / Revised: 22 June 2020 / Accepted: 21 July 2020 / Published online: 3 August 2020

(c) The Author(s) 2020

\begin{abstract}
APOE $\varepsilon 4$ allele is the strongest predictor of Alzheimer's disease (AD) risk, but its role in the association between the deep grey matter volume and cognitive impairment is still unclear. This study investigated the effects of APOE $\varepsilon 4$ allele on this association in non-demented elders. We enrolled 24 patients with mild cognitive impairment (MCI) and 28 normal controls (NC), who underwent the whole brain 3DT $\mathrm{I}$ W MRI scanning, an APOE genotype test, and neuropsychological tests. The right thalamus $(p=0.026)$, the left pallidum $(p=0.026)$, and the bilateral amygdala (left $p=0.042$, right $p=0.048$ ) atrophied in MCI, and their volume were positively correlated with the cognitive scores (MoCA) $(p<0.05)$. Furthermore, the general liner regression model suggested that the correlation between the right thalamus and the putamen volume with MoCA scores was different in the APOE $\varepsilon 4$ carriers and non- carriers. Compared with the non APOE 4 carriers, the right thalamus atrophied more rapidly when the cognition decline in APOE $\varepsilon 4$ carriers, while the right putamen compensatory expansion to slow the rate of cognitive decline although failed. This suggested that the right putamen showed stronger compensation by increasing the volume at the early stage of cognitive impairments in the APOE $\varepsilon 4$ carriers, while this compensatory change had been disappeared in the right thalamus. In conclusion, APOE $\varepsilon 4$ allele modifies the correlation between the right thalamus, the right putamen, and MoCA scores, and it has a potential selective effect on the relationship between cognition and brain structures to some extent in non-demented elders.
\end{abstract}

Keywords APOE 4 allele $\cdot$ Deep grey matter volume $\cdot$ Mild cognitive impairments $\cdot$ Neuropsychological test

Weiping Li and Yu Xie are co-first authors, and have contributed to this work equally.

Zhenyu Yin

zhenyuyin68@163.com

$\triangle$ Bing Zhang

bingzhang4211@163.com

1 Department of Radiology, The Affiliated Drum Tower Hospital of Nanjing University Medical School, Nanjing, China

2 Department of Geriatrics, The Affiliated Drum Tower Hospital of Nanjing University Medical School, Nanjing, China

3 Department of Neurology, The Affiliated Drum Tower of Nanjing University Medical School, Nanjing, China

\section{Introduction}

Apolipoprotein E (APOE) is one of the known common genetic risk factors for Alzheimer's disease (AD), and gene carrying is the strongest predictor of AD risk and has been shown to moderate amyloid-related memory decline in preclinical AD [1,2]. Strong evidence has shown that APOE genotype influences the progression of mild cognitive impairment (MCI) or AD in longitudinal studies, and some case-control studies have demonstrated it to be the risk link to AD [3-5]. Previous studies have demonstrated that APOE $\varepsilon 4$ allele carriers have smaller whole brain volume [6], hippocampus [7, 8], amygdala [9], inferior frontal gyrus cortex [10], and entorhinal cortex [11] than non-carriers in MCI or $\mathrm{AD}$, while there are some controversial announce which suggested that APOE $\varepsilon 4$ have no effect on the whole brain volume, hippocampus [12], amygdala [13], and entorhinal cortex [14] in AD. These controversial observations on the association between APOE $\varepsilon 4$ allele and the deep grey matter 
volume and some cortical regions remain unclear. Besides, these morphological studies mainly conducted using manual or semi-automated techniques and only the region of interest were comprehensive studied. Obviously, an automated measures and overall deep grey matter volume study is needed in providing a thorough view of the effect of APOE $\varepsilon 4$.

Several number of studies have focused on the volume reduction in cortical structures $[15,16]$, and it is widely known that the atrophy of medial temporal structures including the hippocampus and entorhinal cortex can be present in the early stage of $\mathrm{AD}$ and probably amnestic mild cognitive impairment (aMCI), the structural and functional changes of which structures can result in an impairment of episodic memory. While, there are relatively few analysis of the subcortical region such as the basal ganglia and thalamus. Deep grey matter structures are not uniform, which usually include bilateral thalamus, caudate, putamen, pallidum, hippocampus, amygdala, and accumbens. Anatomically, smaller hippocampal volume is correlated with an increased progressive rate from $\mathrm{MCI}$ to $\mathrm{AD}$, which was proved an early sign of $\mathrm{AD}$ pathology [17]. Previous studies showed that these deep grey matter structures are correlated with memory, shifting attention, emotional learning, and spatial working memory [18, 19], which were impaired in AD. Furthermore, some studies found that regional atrophic changes in the hippocampus, thalamus, putamen, and caudate in $\mathrm{AD}$ and even in the MCI [20-22]. While, another study unexpectedly demonstrated larger caudate volume in the AD patient [23]. Moreover, previous studies mostly showed that the AD patients have decreased volume of deep grey matter volume.

Therefore, it is very informative to determine more precisely the relationship between deep grey matter volume and cognitive level both to investigate the pathology of mild cognitive impairments and further to identify the APOE $\varepsilon 4$ allele effects on MCI subjects. However, there are not a lot studies focus on the effect of the APOE $\varepsilon 4$ allele on the association between cognition impairments and the deep grey matter volume in MCI patients. This study used the FreeSurfer automated software package to analyze the deep grey matter volume structural MRI data obtained from normal controls and MCI, and then studied the APOE $\varepsilon 4$ effect on its association with cognition. We hypothesized that the atrophy of the deep grey matter volume would be significantly correlated with cognitive impairments, and this correlation might be modified by the APOE $\varepsilon 4$ gene.

\section{Materials and methods}

\section{Subjects}

A total of 52 participants with $24 \mathrm{MCI}$ and $28 \mathrm{NC}$ were recruited from the Department of Geriatrics of the Affiliated
Drum Tower Hospital of Nanjing University Medical School from March 2015 to June 2018. Written informed consent was obtained from each participant, which was approved by the ethics committee of the Nanjing Drum Tower Hospital, Nanjing, China. Each subject underwent a whole brain MRI scanning, an APOE genotype test, and neuropsychological tests including MMSE, MoCA, ADAScog, ADLn and Digit span, all of which were performed by expert neurologists blinded to the results of MR imaging. All the subjects were right-handed.

Patients with MCI met the clinical criteria established by Petersen and colleagues with criteria as follows: subjective memory complaints, CDR score of 0.5 , and impaired memory function in memory tests. The threshold for memory impairment was derived from the same literature as scoring $>1.5 \mathrm{SD}$ below the mean of age- and education-adjusted norms on memory test. Twenty-eight subjects (NCs) were identified as individuals who (a) had no cognitive complaints, (b) had a normal level of clinical rating scales, and (c) had no evidence of any abnormality determined by the conventional MRI.

The exclusion criteria were set as follows: (1) concurrent illnesses (such as depression, diabetes mellitus, addictions, or psychiatric diseases other than $\mathrm{AD}$ ) or treatments interfering with cognitive function; (2) a score higher than four on the Hachinski Ischemic Scale; (3) the presence of structural abnormalities that could cause cognitive impairment, or the presence of leukoaraiosis higher than Fazekas' grade I, which were identified by the conventional MRI; or (4) not right-handed.

\section{Genotyping}

To identify the APOE genotype, genotyping was conducted using Polymorphic DNA at two loci in APOE exon 4 by amplicon sequencing methods to produce small polymerase chain reaction products that serve as the templates for bidirectional sequencing. Participants with APOE genotypes $2 / 4,3 / 4$, and $4 / 4$ were labeled as APOE $\varepsilon 4$ carriers, whereas other genotypes were labeled as APOE $\varepsilon 4$ non-carriers.

\section{Cognitive assessment}

Neuropsychological assessment was evaluated within 2 weeks after MRI by neuropsychologists. The test battery included the Mini-Mental State Examination (MMSE), The Montreal Cognitive Assessment (MoCA), Alzheimer's Disease Assessment Scale-cognitive subscale (ADAS$\operatorname{cog}$ ), CDR, Digit span total numbers (in order and backward), Activity of Daily Living Scale (ADL), and Hamilton Depression Rating Scale (HAMD). The HAMD tests were used to exclude depression. 


\section{Magnetic resonance imaging acquisition and pre-processsing}

All magnetic resonance imaging scans were performed using a Philips 3.0 T scanner (Achieva 3.0 T TX, Philips Medical Systems, The Netherlands) equipped with an eight-channel phase array head coil. A three-dimensional, high-resolution sagittal T1-weighted scan with turbo fast echo acquisition was performed with repetition time (TR)/echo time (TE)/ inversion time (TI) at 9.8/4.6/900 ms, with a flip angle of $8^{\circ}$ and $1.0 \mathrm{~mm}$ isotropic resolution for anatomical reference. All scans were conducted by an experienced neurologist with the aim of excluding gross brain abnormalities.

Pre-processing of 3D-T1-weighted images were performed using Freesurfer v5.3.0 (https://www.nmr.mgh. harvard.edu/freesurfer/) to segment the deep grey matter volume. First, the high-resolution 3D-T1-weighted images were normalized for intensity and resampled to isotropic voxels of $1 \mathrm{~mm}^{3}$ [24]. Next, the skull was removed using a skull-stripping algorithm and segmented into three tissue types (white matter, grey matter, and CSF). Finally, based on the Desikan-Killiany atlas, deep grey matter volumes named "aseg.stats" files were extracted from FreeSurfer for further statistical analysis.

\section{Statistical analysis}

Descriptive analyses were performed to describe the variables in each group. Categorical variables were compared using the Chi-square test or Fisher's exact test. These analyses were performed using SPSS version 23 with the significant $p$ value less than 0.05 .

In the primary analysis, associations between the cognitive rating scales (dependent variables) and deep grey matter volume (independent variable) were evaluated using a linear regression model (Model 1), adjusted for age, gender, education, and whole brain volume.

In Model 2, we assessed the effects of any differences in the deep grey matter volume on cognitive rating scales above and beyond the effects of the APOE status by adding the APOE status variables [ $\varepsilon 4$ carrier (1) versus non-carrier (0)] to Model 1 as independent variables. If the interaction coefficient was significant at a level of $p<0.05$, it indicated that the association between cognitive rating scales and the difference in deep grey matter volume depended on the APOE $\varepsilon 4$ genes. If the interaction was significant, Model 1 was used for each subgroup (APOE $\varepsilon 4$ carriers and non- APOE $\varepsilon 4$ carriers) separately.

\section{Results}

\section{Demographics and behavioral data}

In this study, 24 subjects fulfilled the criteria of MCI and there were not differences in terms of age, gender, and education between groups. The demographics and neuropsychological tests are listed in Table 1. Patients with MCI had significant lower MMSE, MoCA, ADAScog, digit span (in order), and digit span (backward) than NC.

\section{Analysis of the deep grey matter volume}

For the deep grey matter, we studied 14 deep grey matter (seven per hemisphere). The differences between MCI and NC groups are showed in Table 2, which revealed that the right thalamus ( $p=0.026)$, the left pallidum $(p=0.026)$, and the bilateral amygdala (left $p=0.042$, right $p=0.048$ ) atrophied in MCI.

Table 1 Demographic and clinical characteristic of patients and normal controls

\begin{tabular}{|c|c|c|c|c|}
\hline & $\mathrm{NC}(n=28)$ & $\operatorname{MCI}(n=24)$ & $t / \chi^{2}$ & $p$ \\
\hline $\begin{array}{l}\mathrm{APOE} \varepsilon 4 \text { car- } \\
\text { riers/non } \\
\text { APOE } 4 \text { carri- } \\
\text { ers }(n)\end{array}$ & $3 / 25$ & $7 / 17$ & 0.157 & 0.092 \\
\hline Age (years) & $74.32 \pm 5.64$ & $77.08 \pm 5.30$ & -1.808 & 0.077 \\
\hline \multicolumn{5}{|l|}{ Sex, $n(\%)$} \\
\hline Male & $22(78.6 \%)$ & $17(70.8 \%)$ & 0.413 & 0.541 \\
\hline Female & $6(21.4 \%)$ & $7(29.2 \%)$ & & \\
\hline Education (years) & $14.79 \pm 2.52$ & $13.38 \pm 3.03$ & 1.834 & 0.073 \\
\hline MMSE (score) & $25.97 \pm 2.36$ & $28.93 \pm 0.97$ & 5.299 & $<0.001 * *$ \\
\hline MoCA (score) & $21.81 \pm 2.13$ & $27.43 \pm 2.36$ & 9.032 & $<0.001 * *$ \\
\hline ADAScog & $5.04 \pm 3.31$ & $7.50 \pm 4.43$ & -2.292 & $0.026 *$ \\
\hline ADL & $21.29 \pm 5.33$ & $22.83 \pm 6.44$ & -0.948 & 0.348 \\
\hline $\begin{array}{l}\text { Digit span (in } \\
\text { order) }\end{array}$ & $8.11 \pm 1.39$ & $7.25 \pm 1.35$ & 2.233 & $\mathbf{0 . 0 3 0} *$ \\
\hline $\begin{array}{l}\text { Digit span (back- } \\
\text { ward) }\end{array}$ & $5.29 \pm 1.43$ & $3.67 \pm 0.76$ & 4.953 & $<0.001 * *$ \\
\hline
\end{tabular}

The significant difference marked bold

Data are presented as means \pm standard deviations

$M C I$ mild cognitive impairment, $N C$ normal control; $E d u$ education, MMSE Mini-Mental State Examination, MoCA Montreal Cognitive Assessment Beijing Version, ADAScog Alzheimer's Disease Assessment Scale-cognitive subscale, $A D L$ Activity of Daily Living Scale

$*$ Significant difference with a $p$ value $<0.05 ; * *$ Significant difference with a $p$ value $<0.01$ 
Table 2 The volume of deep grey matter between MCI patients and normal control participants

\begin{tabular}{lcccc}
\hline & NC $(n=28)$ & MCI $(n=24)$ & $t$ & $p$ \\
\hline Thalamus $\left(\mathrm{mm}^{3}\right)$ & & & & \\
R & $5675.69 \pm 608.63$ & $5254.07 \pm 712.47$ & 2.302 & $\mathbf{0 . 0 2 6}^{*}$ \\
L & $5802.62 \pm 588.63$ & $5537.84 \pm 542.88$ & 1.676 & 0.100 \\
Caudate $\left(\mathrm{mm}^{3}\right)$ & & & & \\
R & $3597.31 \pm 523.90$ & $3518.92 \pm 614.21$ & 0.497 & 0.621 \\
L & $3350.18 \pm 609.82$ & $3189.35 \pm 493.92$ & 1.033 & 0.306 \\
Putamen $\left(\mathrm{mm}^{3}\right)$ & & & & \\
R & $5297.18 \pm 700.38$ & $4901.68 \pm 932.15$ & 1.744 & 0.087 \\
L & $5417.30 \pm 889.15$ & $4984.41 \pm 928.61$ & 1.715 & 0.093 \\
Pallidum $\left(\mathrm{mm}^{3}\right)$ & & & & \\
R & $1405.46 \pm 217.38$ & $1374.34 \pm 272.11$ & 0.458 & 0.649 \\
L & $1497.53 \pm 163.09$ & $1374.66 \pm 221.05$ & 2.301 & $\mathbf{0 . 0 2 6}$ \\
Hippocampus $\left(\mathrm{mm}^{3}\right)$ & & & & \\
R & $3409.86 \pm 994.49$ & $3241.47 \pm 609.26$ & 0.721 & 0.474 \\
L & $3209.29 \pm 824.66$ & $3033.99 \pm 609.98$ & 0.859 & 0.395 \\
Amygdala $\left(\mathrm{mm}^{3}\right)$ & & & & \\
R & $1776.55 \pm 543.39$ & $1495.37 \pm 405.19$ & 2.085 & $\mathbf{0 . 0 4 2 *}$ \\
L & $1486.77 \pm 221.92$ & $1332.13 \pm 324.11$ & 2.031 & $\mathbf{0 . 0 4 8 *}$ \\
Accumbens $\left(\mathrm{mm}^{3}\right)$ & & & & \\
R & $510.56 \pm 101.74$ & $506.15 \pm 99.84$ & 0.157 & 0.876 \\
L & $533.35 \pm 143.47$ & $489.95 \pm 118.81$ & 1.176 & 0.245 \\
Whole brain $\left(\mathrm{cm}^{3}\right)$ & $1409.27 \pm 291.83$ & $1295.72 \pm 239.76$ & 1.517 & 0.136 \\
\hline
\end{tabular}

The significant difference marked bold

Data are presented as means \pm standard deviations

$R$ right side, $L$ left side

*Significant difference with a $p$ value $<0.05$ with independent-samples $T$ test. The $p$ value in Levene's test for equality of variances is 0.2

\section{Association between the deep grey matter volume and neuropsychological test scores}

The general liner regression analysis between the deep grey matter volume and neuropsychological tests scores is showed in Table 3 (model 1) with age, gender, education, and whole brain volume as covariates. There was no correlation between MMSE, ADL, ADAScog, and the deep grey matter volume. The MoCA scores was positive correlated with the right thalamus $(\beta=0.360, p=0.006)$, putamen volume $(\beta=0.356, p=0.006)$, the left pallidum $(\beta=0.366$, $p=0.012$ ), and bilateral amygdala volume (right $\beta=0.387$, $p=0.005$ and left $\beta=0.436, p=0.006$ ). The digit span (in order) scores was positive associated with the right thalamus volume $(\beta=0.258, p=0.043)$. In addition, the digit span (backward) scores were positive correlated with the bilateral thalamus volume (right $\beta=0.462, p=0.001$ and left $\beta=0.411, p=0.003$ ) and caudate volume (right $\beta=0.356$, $p=0.015$ and left $\beta=0.311, p=0.023$ ).

\section{Apoes4 allele effects on the correlation of deep grey matter volume and neuropsychological test scores}

To assess whether APOE 4 modifies the significant association between cognition and deep grey matter volume, we added the APOE genotype [ $\varepsilon 4$ carrier (1) versus non-carrier (0)] to Model 1. When the correlation coefficient was significant at a level of $p<0.05$, it was concluded that the association was modified by APOE $\varepsilon 4$ genes.

The differences of the correlation between the right thalamus volume and MOCA scores in APOE $\varepsilon 4$ allele carriers and non-carriers reached the statistics difference $(B=-0.534, p=0.034)$ (Table 4$)$. In addition, the association of the right putamen volume and MOCA scores in APOE 4 allele carriers and non-carriers was also different $(B=-38.686, p=0.014)$. There are no statistics differences between other deep grey matter volume and the neuropsychological test scores with the APOE $\varepsilon 4$ allele into consideration. 


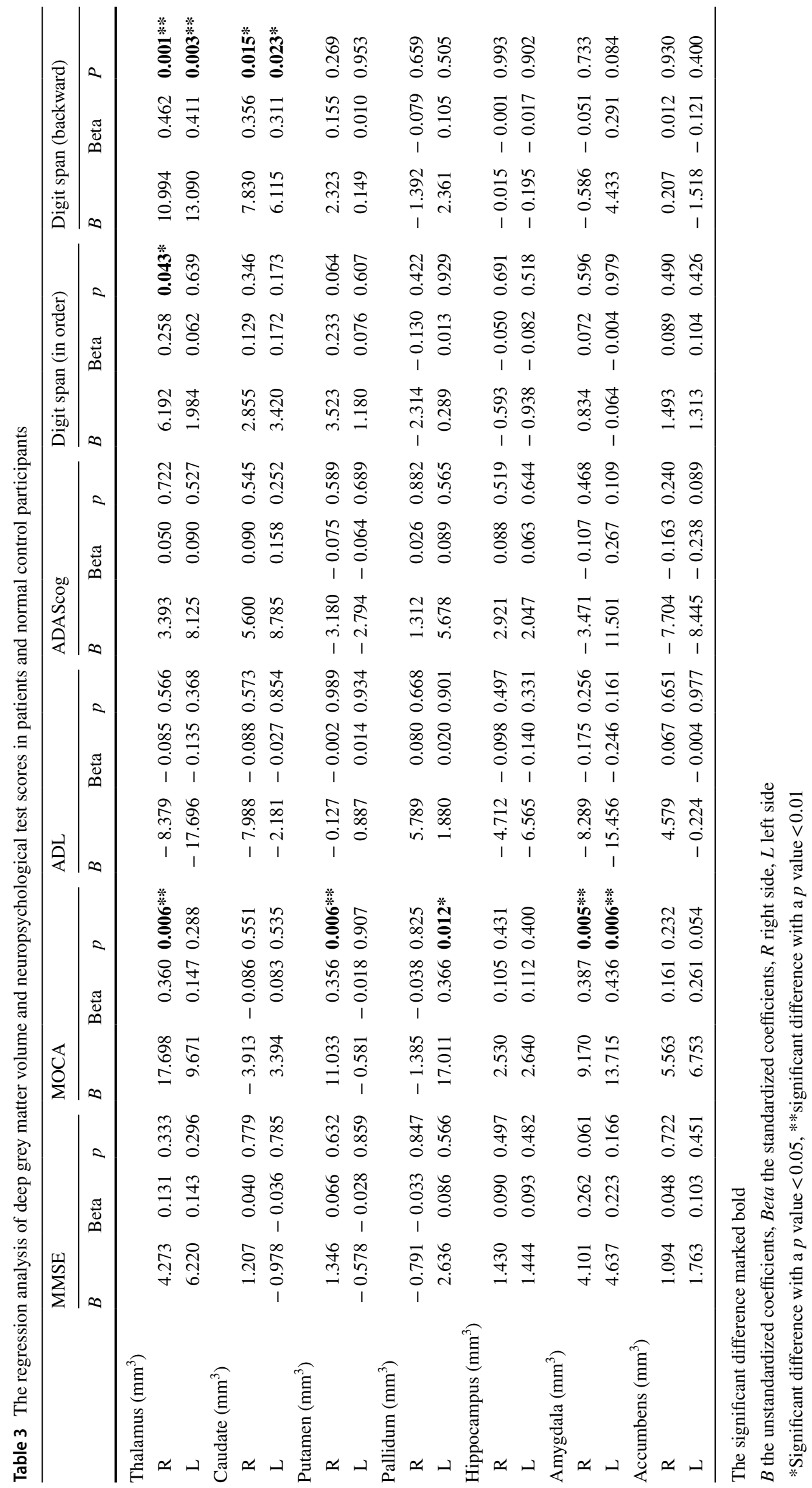




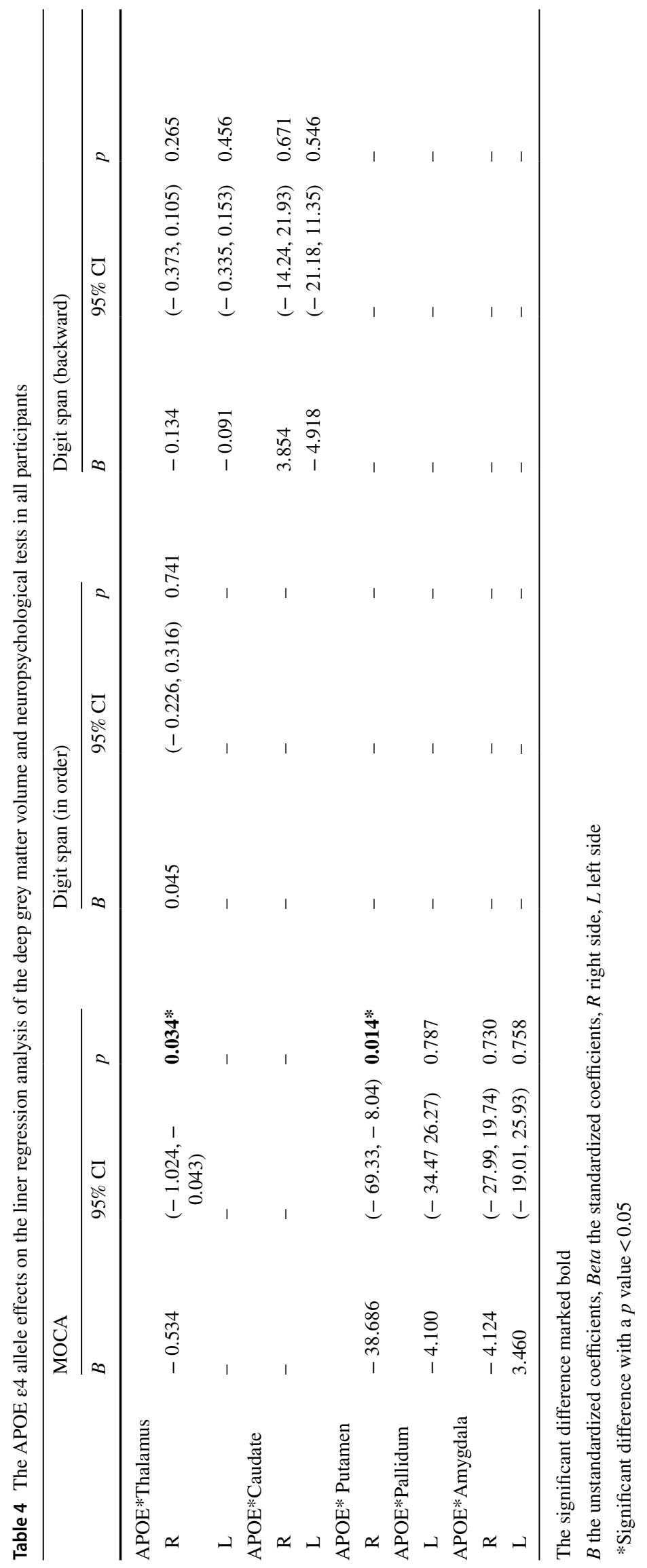


Fig. 1 Effect of APOE $\varepsilon 4$ on the correlation between the subcortical volume and MoCA score. In the APOE $\varepsilon 4$ group, the participants with poor cognition have bigger right putamen volume and smaller right thalamus volume
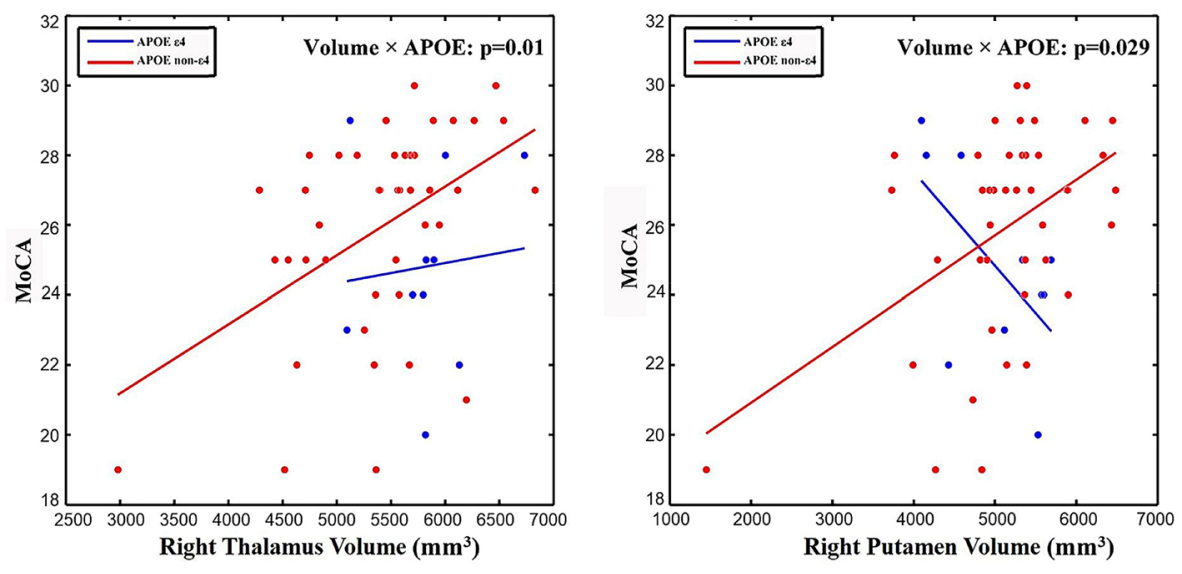

In the APOE $\varepsilon 4$ group, the participants with poor MoCA scores have bigger right putamen volume and smaller right thalamus volume (Fig. 1). The unstandardized coefficients in the regression model of the right thalamus and MoCA scores in APOE $\varepsilon 4$ allele carriers were bigger than in the APOE $\varepsilon 4$ non-carriers (Table 5).

\section{Discussion}

In this study, we explored the APOE e4 allele effects on the correlation between deep grey matter structure volume and cognitive impairment in non-demented elders. As expected, the bilateral thalamus, caudate and amygdala, the right putamen, and the left pallidum atrophied in MCI and their volume were positively associated with cognitive level. Furthermore, taking the APOE $\varepsilon 4$ allele effects into account, the relationship of the right thalamus volume, the right putamen volume, and MoCA scores weakened in MCI and normal controls. In the APOE $\varepsilon 4$ group, the participants with poor cognitive performance have bigger right putamen volume and smaller right thalamus volume. And for every point decreased in the MoCA score, the right thalamus volume decreased more in the APOE $\varepsilon 4$ carriers group than that in the APOE non- $\varepsilon 4$ carriers group, which indicate a potential selective effect on the atrophy of the deep grey matter by the APOE $\varepsilon 4$ allele.

The thalamus is a large mass of grey matter located in the forebrain, which is manifold connected to the hippocampus via the mammillo-thalamic tract, and this tract comprises the mammillary bodies and fornix [25]. This nucleus has multiple functions, such as relaying of sensory signals, including motor signals, to the cerebral cortex, and the regulation of consciousness, sleep, and alertness [26]. It is functionally connected to the hippocampus as part of the extended hippocampal system at the thalamic anterior nuclei with respect to spatial memory and spatial sensory datum, and they are crucial for human episodic memory and rodent event memory [27-29]. The medial temporal lobe connects to the thalamus and retrosplenial cortex, which is part of the hippocampal-diencephalic system, and this structure is very important for the normal episodic memory [30], so it is expected to detect memory impairment in patient with damage in these structures, such as thalamus. The thalamus, the medial temporal structural, and the hippocampus connect each other somehow in different ways. We found that the right thalamus volume was correlated with the MoCA scores and the digit span (both in order and backward) scores
Table 5 The correlation between MOCA and the right thalamus and putamen in APOE $\varepsilon 4$ allele carriers and noncarriers

\begin{tabular}{|c|c|c|c|c|c|c|}
\hline & \multicolumn{3}{|c|}{ APOE4 carriers } & \multicolumn{3}{|c|}{ Non-APOE4 carriers } \\
\hline & \multicolumn{3}{|l|}{ MOCA } & \multicolumn{3}{|l|}{ MOCA } \\
\hline & $B$ & Beta & $p$ & $B$ & Beta & $p$ \\
\hline \multicolumn{7}{|c|}{ Thalamus } \\
\hline $\mathrm{R}$ & 55.255 & 0.681 & 0.209 & 22.251 & 0.473 & $0.001 * *$ \\
\hline \multicolumn{7}{|c|}{ Putamen } \\
\hline $\mathrm{R}$ & -50.724 & -1.003 & 0.257 & 12.761 & 0.442 & $0.001 * *$ \\
\hline
\end{tabular}

The significant difference marked bold

$B$ the unstandardized coefficients, Bet $a$ the standardized coefficients, $R$ right side

**Significant difference with a $p$ value $<0.01$ 
in the participant. The left thalamus volume was positively correlated with the digit span (backward) scores in the participant. The previous study suggested that digit span (in order) is a measure of short-term memory, whereas digit span (backward) is a measure of working memory, which was the prevalence view in clinical practice [31]. Therefore, in this research, smaller bilateral thalamus volume was associated with worse working memory, and right thalamus was associated with worse short-term memory, and immediate and delayed recall.

Our findings were consistent with our previous hypothesis that APOE e4 carrier status has effect on the correlation between the thalamus, putamen volume, and the cognitive level. Furthermore, when the cognition decline in APOE $\varepsilon 4$ carriers, the right putamen compensatory expansion to slow the rate of cognitive decline with the effect of the APOE $\varepsilon 4$ allele. While, the right thalamus atrophied more rapidly in the APOE $\varepsilon 4$ carriers. Our estimates of APOE e 4 allele carriers risk expand and extend the previous related studies and provide new insights into the MCI.

Most MRI-based studies have found that the hippocampus is among the first brain structures to be affected by $\mathrm{AD}$, the author even found that pre-MCI subjects showed lower left hippocampal volume at baseline than the normal controls in a longitudinal study [32]. Also, there are many studies which have explored the brain atrophy mainly in medial temporal areas in $\mathrm{AD}$ [33] and the atrophy of both bilateral thalamus [34]. Most of these areas were associated with cognitive functions, for example, episodic memories, working memories, languages, and visuospatial skills. Our finding also confirmed such areas atrophy in the right thalamus, the left pallidum, and the bilateral amygdala in MCI patients among all the seven pairs of deep grey matter structures. In our research, comparing MCI with normal controls, we did not find a significant volume reduction in bilateral hippocampus, which might account for the following factors. As we known, hippocampus can be parceled into several subfields, and CA1 was proved to be the earliest and most vulnerable among all subfields in AD-related neuronal loss [35]. We did not parcel the subfields of each grey matter, which may lead to no significant difference in the group comparison of hippocampus as a whole. Also, the MCI subjects in this study was not differentiate whether suffering from the amnestic and non-amnestic domain. Researches have shown that nonamnestic MCI patients had normal hippocampal volume [36], so it is reasonable that we did not find hippocampal atrophy if the MCI subtypes were mixed in our cohort. In the future study, the difference of subcortical volume between amnestic and non-amnestic MCI should be compared.

The striatum usually include the following nucleus, caudate, putamen, and pallidum, which can be divided into the following three function areas: the dorsomedial region (mainly caudate nucleus), dorsolateral area (mainly putamen), and the ventral region [37]. The dorsolateral areas receive information from sensory and motor cortical, which might predict specific executive function and movement skill. The dorsomedial areas receive information from the anterior cingulate and have relationship with specific $\operatorname{cog}$ nitive function. The ventral region receives the projection from hippocampal zone [38]. The striatum has structural and functional connections with the specific cortical areas, which is equivalent to the central point. Therefore, the structure is very sensitive to the pathological changes of neurodegenerative diseases [39]. The previous study showed that beta amyloid can deposited in the striatum in normal cognitive elders,furthermore, the structure and functional connection was changed between the striatum and other brain region in mild cognitive impairment and AD patients [40, 41]. We found that the right putamen volume, the left pallidum, and bilateral amygdala volume were positively correlated with the MoCA scores, and the caudate volume was positive correlated with the digit span (backward) scores. This demonstrated that the caudate was related to the working memory in MCI patients, and the right putamen, the left pallidum, and bilateral amygdala were involved in immediate and delayed recall. A resting-MRI study found that the reduction of the amplitude of low frequency fluctuation (ALFF) in putamen was associated with beta amyloid deposition in MCI patients [42], and this may provide evidence to approve the correlation and the component response which we found. In addition, APOE $\varepsilon 4$ allele had affect the relationship between the right putamen and MoCA scores in this study.

There were several limitations in our study: first, because of the small sample size of APOE $\varepsilon 4$ allele carriers which we recruited, it was difficult to make some correction on the results. In addition, the unstandardized coefficients in the liner regression of the right thalamus volume and the MoCA scores in APOE $\varepsilon 4$ allele carriers were bigger than in the non-carriers. Which suggest that with poorer MoCA score, the right thalamus atrophied more rapidly in the APOE $\varepsilon 4$ carriers than that in the non-carriers, though it did not reach the statistic level and this needs to be further investigated in large sample studies. Second, AD is not a monogenic disease, but probably a polygenetic disease influenced by multiple genes with small or medium risks; in this study, we only detected the APOE genotype and further study is needed to detect the effect of more AD-related gene. Third, this is a cross-sectional study, which was difficult to judge the atrophy order of the deep grey matter structure and ADrelated brain atrophy, such as the entorhinal cortex; furthermore, it is difficult to comprehensively explore on the cognition and deep grey matter. At the same time, because of the cross-sectional study, we did not found the compensatory expansion in the right thalamus at this point, but this does not mean that it does not exist in the earlier point. Therefore, 
follow-up studies that patients compared themselves over time should be conduct to verify some results and generally perfect this APOE $\varepsilon 4$ allele effect in the future.

In summary, we emphasize the importance of APOE e4 allele and identify its effects on the deep grey matter nucleus and cognitive level. The right thalamus atrophied more rapidly when the cognition decline in APOE $\varepsilon 4$ carriers, while the right putamen compensatory expansion to slow the rate of cognitive decline although failed. This suggested that the right putamen showed stronger compensation by increasing the volume at the early stage of cognitive impairments in the APOE $\varepsilon 4$ carriers, while this compensatory change had been disappeared in the right thalamus. Therefore, cognitionrelated APOE e4 risk could provide stronger opportunities by the atrophy of deep grey matter to identify mild cognitive disease. These results may enrich the study on neurodevelopmental alterations, which can provide new insights into the APOE e4 allele and deep grey matter nucleus in the progression of the MCI.

Acknowledgements This work was supported by the National Natural Science Foundation of China (81720108022, 91649116); the Social Development Project of Science and Technology Project in Jiangsu Province (BE2016605, BE201707); Key Medical Talents of the Jiangsu Province, the "13th Five-Year" Health Promotion Project Of the Jiangsu province (B.Z.2016-2020). The funders had no role in the study design, data collection and analysis, decision to publish, or preparation of the manuscript.

\section{Compliance with ethical standards}

Conflict of interest The authors have no conflict of interest to report.

Open Access This article is licensed under a Creative Commons Attribution 4.0 International License, which permits use, sharing, adaptation, distribution and reproduction in any medium or format, as long as you give appropriate credit to the original author(s) and the source, provide a link to the Creative Commons licence, and indicate if changes were made. The images or other third party material in this article are included in the article's Creative Commons licence, unless indicated otherwise in a credit line to the material. If material is not included in the article's Creative Commons licence and your intended use is not permitted by statutory regulation or exceeds the permitted use, you will need to obtain permission directly from the copyright holder. To view a copy of this licence, visit http://creativecommons.org/licenses/by/4.0/.

\section{References}

1. Farrer LA, Cupples LA, Haines JL, et al. Effects of age, sex, and ethnicity on the association between apolipoprotein E genotype and Alzheimer disease. A meta-analysis. APOE and Alzheimer Disease Meta Analysis Consortium. JAMA. 1997;278:1349-56.

2. Lim YY, Villemagne VL, Pietrzak RH, et al. APOE epsilon4 moderates amyloid-related memory decline in preclinical Alzheimer's disease. Neurobiol Aging. 2015;36:1239-44.

3. Zheng L, Kong X, Cui Y, Wei Y, Zhang J, Wei W. Conversion from MCI to $\mathrm{AD}$ in patients with the APOE $\varepsilon 4$ genotype: prediction by plasma HCY and serum BDNF. Neurosci Lett. 2016;626:19-24.

4. Leonenko G, Shoai M, Bellou E, et al. Genetic risk for alzheimer disease is distinct from genetic risk for amyloid deposition. Ann Neurol. 2019;86:427-35.

5. Oveisgharan S, Buchman AS, Yu L, et al. APOE $\varepsilon 2 \varepsilon 4$ genotype, incident $\mathrm{AD}$ and $\mathrm{MCI}$, cognitive decline, and $\mathrm{AD}$ pathology in older adults. Neurology. 2018;90:e2127-e21342134.

6. Espeseth T, Greenwood PM, Reinvang I, et al. Interactive effects of APOE and CHRNA4 on attention and white matter volume in healthy middle-aged and older adults. Cogn Affect Behav Neurosci. 2006;6:31-433.

7. den Heijer T, Oudkerk M, Launer LJ, van Duijn CM, Hofman A, Breteler MM. Hippocampal, amygdalar, and global brain atrophy in different apolipoprotein E genotypes. Neurology. 2002;59:746-8.

8. Lind J, Larsson A, Persson J, et al. Reduced hippocampal volume in non-demented carriers of the apolipoprotein E epsilon4: relation to chronological age and recognition memory. Neurosci Lett. 2006;396:23-7.

9. Lupton MK, Strike L, Hansell NK, et al. The effect of increased genetic risk for Alzheimer's disease on hippocampal and amygdala volume. Neurobiol Aging. 2016;40:68-77.

10. Hamalainen A, Grau-Olivares M, Tervo S, et al. Apolipoprotein E epsilon 4 allele is associated with increased atrophy in progressive mild cognitive impairment: a voxel-based morphometric study. Neurodegener Dis. 2008;5:186-9.

11. Shaw P, Lerch JP, Pruessner JC, et al. Cortical morphology in children and adolescents with different apolipoprotein $\mathrm{E}$ gene polymorphisms: an observational study. Lancet Neurol. 2007;6:494-500.

12. Drzezga A, Grimmer T, Henriksen G, et al. Effect of APOE genotype on amyloid plaque load and gray matter volume in Alzheimer disease. Neurology. 2009;72:1487-94.

13. Barber R, Ballard C, McKeith IG, Gholkar A, O'Brien JT. MRI volumetric study of dementia with Lewy bodies: a comparison with AD and vascular dementia. Neurology. 2000;54:1304-9.

14. Du AT, Schuff N, Chao LL, et al. Age effects on atrophy rates of entorhinal cortex and hippocampus. Neurobiol Aging. 2006;27:733-40.

15. Bae $S$, Lee $S$, Harada $K$, et al. Engagement in lifestyle activities is associated with increased Alzheimer's disease-associated cortical thickness and cognitive performance in older adults. J Clin Med. 2020;9(5):1424. https://doi.org/10.3390/jcm9051424.

16. Parker TD, Cash DM, Lane CA, et al. Amyloid beta influences the relationship between cortical thickness and vascular load. Alzheimers Dement (Amst). 2020;12:e12022.

17. Ten Kate M, Barkhof F, Boccardi M, et al. Clinical validity of medial temporal atrophy as a biomarker for Alzheimer's disease in the context of a structured 5-phase development framework. Neurobiol Aging. 2017;52(167-182):e161.

18. Lanciego JL, Luquin N, Obeso JA. Functional neuroanatomy of the basal ganglia. Cold Spring Harb Perspect Med. 2012;2:a009621.

19. Herrero MT, Barcia C, Navarro JM. Functional anatomy of thalamus and basal ganglia. Childs Nerv Syst. 2002;18:386-404.

20. de Jong LW, van der Hiele K, Veer IM, et al. Strongly reduced volumes of putamen and thalamus in Alzheimer's disease: an MRI study. Brain. 2008;131:3277-85.

21. Barnes J, Bartlett JW, van de Pol LA, et al. A meta-analysis of hippocampal atrophy rates in Alzheimer's disease. Neurobiol Aging. 2009;30:1711-23.

22. Cho H, Kim JH, Kim C, et al. Shape changes of the basal ganglia and thalamus in Alzheimer's disease: a three-year longitudinal study. J Alzheimers Dis. 2014;40:285-95. 
23. Persson K, Bohbot VD, Bogdanovic N, Selbaek G, Braekhus A, Engedal K. Finding of increased caudate nucleus in patients with Alzheimer'sdisease. Acta Neurol Scand. 2018;137(2):224-32. https://doi.org/10.1111/ane.12800.

24. Fischl B, Salat DH, van der Kouwe AJ, et al. Sequence-independent segmentation of magnetic resonance images. Neuroimage. 2004;23(Suppl 1):S69-84.

25. Carlesimo GA, Lombardi MG, Caltagirone C. Vascular thalamic amnesia: a reappraisal. Neuropsychologia. 2011;49:777-89.

26. Lee CC. Exploring functions for the non-lemniscal auditory thalamus. Front Neural Circuits. 2015;9:69.

27. Stein T, Moritz C, Quigley M, Cordes D, Haughton V, Meyerand E. Functional connectivity in the thalamus and hippocampus studied with functional MR imaging. AJNR Am J Neuroradiol. 2000;21:1397-401.

28. Aggleton JP, Brown MW. Episodic memory, amnesia, and the hippocampal-anterior thalamic axis. Behav Brain Sci. 1999;22:42544 (discussion 444-489).

29. Aggleton JP, O’Mara SM, Vann SD, Wright NF, Tsanov M, Erichsen JT. Hippocampal-anterior thalamic pathways for memory: uncovering a network of direct and indirect actions. Eur J Neurosci. 2010;31:2292-307.

30. Opitz B, Friederici AD. Interactions of the hippocampal system and the prefrontal cortex in learning language-like rules. Neuroimage. 2003;19:1730-7.

31. Luo D, Chen G, Zen F, Murray B. Modeling working memory tasks on the item level. Intelligence. 2010;38:66-82.

32. Duara R, Loewenstein DA, Greig MT, et al. Pre-MCI and MCI: neuropsychological, clinical, and imaging features and progression rates. Am J Geriatr Psychiatry. 2011;19:951-60.

33. Karas GB, Scheltens P, Rombouts SA, et al. Global and local gray matter loss in mild cognitive impairment and Alzheimer's disease. Neuroimage. 2004;23:708-16.
34. Pedro T, Weiler M, Yasuda CL, et al. Volumetric brain changes in thalamus, corpus callosum and medial temporal structures: mild Alzheimer's disease compared with amnestic mild cognitive impairment. Dement Geriatr Cogn Disord. 2012;34:149-55.

35. West MJ, Kawas CH, Stewart WF, Rudow GL, Troncoso JC. Hippocampal neurons in pre-clinical Alzheimer's disease. Neurobiol Aging. 2004;25:1205-12.

36. Kantarci K, Petersen RC, Przybelski SA, et al. Hippocampal volumes, proton magnetic resonance spectroscopy metabolites, and cerebrovascular disease in mild cognitive impairment subtypes. Arch Neurol. 2008;65:1621-8.

37. Voorn P, Vanderschuren LJ, Groenewegen HJ, Robbins TW, Pennartz CM. Putting a spin on the dorsal-ventral divide of the striatum. Trends Neurosci. 2004;27:468-74.

38. O'Mara SM, Sanchez-Vives MV, Brotons-Mas JR, O'Hare E. Roles for the subiculum in spatial information processing, memory, motivation and the temporal control of behaviour. Prog Neuropsychopharmacol Biol Psychiatry. 2009;33:782-90.

39. Cole MW, Pathak S, Schneider W. Identifying the brain's most globally connected regions. Neuroimage. 2010;49:3132-48.

40. Rodriguez-Vieitez E, Saint-Aubert L, Carter SF, et al. Diverging longitudinal changes in astrocytosis and amyloid PET in autosomal dominant Alzheimer's disease. Brain. 2016;139:922-36.

41. Han SD, Arfanakis K, Fleischman DA, et al. Functional connectivity variations in mild cognitive impairment: associations with cognitive function. J Int Neuropsychol Soc. 2012;18:39-48.

42. Ren P, Lo RY, Chapman BP, Mapstone M, Porsteinsson A, Lin F. Longitudinal alteration of intrinsic brain activity in the striatum in mild cognitive impairment. J Alzheimers Dis. 2016;54:69-78.

Publisher's Note Springer Nature remains neutral with regard to jurisdictional claims in published maps and institutional affiliations. 